\title{
Article
}

\section{Dysphagia screening and risks of pneumonia and adverse outcomes after acute stroke: An international multicenter study}

Ouyang, Menglu, Boaden, Elizabeth, Arima, Hisatomi, Lavados, Pablo M., Billot, Laurent, Hackett, Maree, Olavarría, Verónica V., Muñoz-Venturelli, Paula, Song, Lili, Rogers, Kris, Middleton, Sandy, Pontes-Neto, Octavio M., Lee, Tsong-Hai, Watkins, Caroline Leigh, Robinson, Thompson and Anderson, Craig S.

Available at http://clok.uclan.ac.uk/28949/

Ouyang, Menglu, Boaden, Elizabeth ORCID: 0000-0002-4647-6392, Arima, Hisatomi, Lavados, Pablo M., Billot, Laurent, Hackett, Maree ORCID: 00000003-1211-9087, Olavarría, Verónica V., Muñoz-Venturelli, Paula, Song, Lili et al (2020) Dysphagia screening and risks of pneumonia and adverse outcomes after acute stroke: An international multicenter study. International Journal of Stroke, 15 (2). pp. 206-215. ISSN 1747-4930

It is advisable to refer to the publisher's version if you intend to cite from the work. http://dx.doi.org/10.1177/1747493019858778

For more information about UCLan's research in this area go to http://www.uclan.ac.uk/researchgroups/ and search for <name of research Group>.

For information about Research generally at UCLan please go to http://www.uclan.ac.uk/research/

All outputs in CLoK are protected by Intellectual Property Rights law, including Copyright law. Copyright, IPR and Moral Rights for the works on this site are retained by the individual authors and/or other copyright owners. Terms and conditions for use of this material are defined in the policies page. 


\section{Research}

\section{Corresponding Author}

Professor Craig S Anderson

The George Institute for Global Health

PO Box M201, Missenden Road, NSW 2050, AUSTRALIA

T: +61-2-9993-4500; F: +61-2-9993-4502; E: canderson@georgeinstitute.org.au

\section{Dysphagia screening and risks of pneumonia and adverse outcomes after acute stroke:} an international multicentre study

Menglu Ouyang MPH, ${ }^{1,2}$ Elizabeth Bowden PhD, ${ }^{3}$ Hisatomi Arima MD PhD, ${ }^{1,4}$ Pablo M.

Lavados MD MPH, ${ }^{5,6,7}$ Laurent Billot MRes, ${ }^{1}$ Maree L. Hackett PhD, ${ }^{1,3}$ Verónica V.

Olavarría MD MS., ${ }^{5,6}$ Paula Muñoz-Venturelli MD PhD, , ${ }^{1,5,6}$ Lili Song MD PhD, ${ }^{1,2,}$ Kris

Rogers MBiostat PhD, ${ }^{1}$ Sandy Middleton $\mathrm{PhD},{ }^{8}$ Octavio M. Pontes-Neto MD PhD, ${ }^{9}$ Tsong-

Hai Lee MD PhD, ${ }^{10}$ Caroline Watkins PhD, ${ }^{3,8}$ Thompson Robinson MD, ${ }^{11}$ and Craig S.

Anderson MD PhD; ${ }^{1,2,12}$ for the HeadPoST Investigators*

${ }^{1}$ The George Institute for Global Health, Faculty of Medicine, University of New South Wales, Sydney, Australia

${ }^{2}$ The George Institute China at Peking University Health Science Center, Beijing, China

${ }^{3}$ College of Health and Wellbeing, University of Central Lancashire, Preston, Lancashire,

${ }^{4}$ Department of Preventive Medicine and Public Health, Faculty of Medicine, Fukuoka University, Fukuoka, Japan;

${ }^{5}$ Department of Neurology and Psychiatry, Clínica Alemana de Santiago, Santiago, Chile

${ }^{6}$ Facultad de Medicina, Clinica Alemana Universidad del Desarrollo, Santiago, Chile

${ }^{7}$ Departamento de Ciencias neurológicas, Facultad de medicina, Universidad de Chile Universidad de Chile, Santiago, Chile;

${ }^{8}$ Nursing Research Institute, St Vincent’s Health (Sydney) Australia, Australian Catholic University, Sydney, Australia

${ }^{9}$ Stroke Service - Neurology Division, Department of Neuroscience and Behavioral Sciences, Ribeirão Preto Medical School, University of Sao Paulo, Ribeirão Preto - SP, Brazil

${ }^{10}$ Stroke Center and Department of Neurology, Linkou Chang Gung Memorial Hospital and College of Medicine, Chang Gung University, Taoyuan, Taiwan

${ }^{11}$ Department of Cardiovascular Sciences and NIHR Leicester Biomedical Research Centre, University of Leicester, Leicester, UK

${ }^{12}$ Neurology Department, Royal Prince Alfred Hospital, Sydney Health Partners, Sydney, Australia 
*full list of investigators listed in the Supplementary Appendix

Word Count: Abstract 253; body 3173

\section{Tables: 2}

Figures: 2

Supplemental tables: 16

\section{Supplemental figures: 13}

Number of reference: 23

Key words: dysphagia, screen, assessment, acute stroke, pneumonia, disability, clinical trial 


\begin{abstract}
Background: Dysphagia is associated with aspiration pneumonia after stroke. Data are limited on the influences of dysphagia screen and assessment in clinical practice.
\end{abstract}

Aims: To determine associations between a 'brief' screen and 'detailed' assessment of dysphagia on clinical outcomes in acute stroke patients.

Methods: A prospective cohort study analysed retrospectively using data from a multi-centre, cluster cross-over, randomised controlled trial (Head Positioning in Acute Stroke Trial [HeadPoST]) from 114 hospitals in nine countries. HeadPoST included 11,093 acute stroke patients randomised to lying-flat or sitting-up head positioning. Herein, we report pre-defined secondary analyses of the association of dysphagia screening and assessment, and clinical outcomes of pneumonia and death or disability (modified Rankin scale 3-6) at 90 days.

Results: Overall, 8784 (79.2\%) and 3917 (35.3\%) patients were screened and assessed for dysphagia, respectively, but the frequency and timing for each varied widely across regions. Neither use of a screen nor an assessment for dysphagia were associated with the outcomes, but their results were: compared to 'screen-pass' patients, those who failed had higher risks of pneumonia (adjusted odds ratio[aOR] 3.00, 95\% confidence interval[CI] 2.18-4.10) and death or disability (aOR 1.66, 95\%CI 1.41-1.95). Similar results were evidence for the results of an assessment for dysphagia. Subsequent feeding restrictions were related to higher risk of pneumonia in patients failed dysphagia screen or assessment (aOR 4.06, 95\%CI 1.72-9.54).

Conclusions: Failing a dysphagia screen is associated with increased risks of pneumonia and poor clinical outcome after acute stroke. Further studies concentrate on determining the effective subsequent feeding actions are needed to improve patient outcomes. 


\section{Introduction}

Pneumonia is a common complication of acute stroke, which increases the likelihood of death and poor recovery, and costs of care. ${ }^{1-3}$ As dysphagia is also common and associated with aspiration pneumonia, ${ }^{4,5,6}$ stroke management guidelines recommend that health professionals screen for this impairment before their patients receive any oral intake of food, fluid or medications..$^{7-12}$ However, the evidence base supporting this recommendation is of moderate grade, with only one cluster clinical trial of implementing protocols that included dysphagia screening/assessment in conjunction with fever and hyperglycaemia management showing improved short and long-term clinical outcomes. ${ }^{13}$ A small 'before-and-after' study showed that the implementation of dysphagia screening by nurses reduced pneumonia and length of stay in hospital, ${ }^{14}$ while delays in screening and assessment for dysphagia in patients were associated with pneumonia in the UK national stroke registry. ${ }^{15}$ Although a range of simple and systematic approaches exist for the assessment of dysphagia, data are limited on how well these are incorporated into clinical pathways and influence feeding actions and clinical outcomes in practice. ${ }^{14}$ The international Head Positioning in Acute Stroke Trial (HeadPoST) dataset, ${ }^{16}$ therefore, provides an opportunity to examine the utility of screening and assessment of dysphagia, and feeding actions, on key clinical outcomes in a large cohort of stroke patients with a broad range of characteristics who were recruited from 114 hospitals in nine countries.

\section{Methods}

\section{Design}

This study is a pre-defined secondary analysis of HeadPoST, the design and main results of which are outlined in detail elsewhere. ${ }^{16}$ In brief, HeadPoST was an international, multicentre, cluster cross-over, randomised controlled trial of two different head positions in 11,093 adult patients with acute stroke undertaken between March 2015 and November 2016. Patients were 
excluded if they had resolved neurological symptoms consistent with a transient ischaemic attack, a clear contraindication to either head position, any medical condition that would compromise adherence to the protocol or assigned head position, or refusal to participate. The main results showed there were no significant differences in disability outcomes and risks between those assigned to the lying-flat or sitting-up head positions for at least 24 hours after hospital admission. The study was approved by ethics committees of participating sites. Consent was obtained from all patients or appropriate surrogates for participation, use of medical data, and central follow-up assessment.

\section{Assessments}

After central randomisation, stratified by country, centres were required to implement the first assigned intervention position until a target number of consecutive patients was reached, before crossing over to apply the other intervention to a similar number of consecutive patients. Data collection included the time, result and action of any: dysphagia screen, defined as use of a simple brief non-invasive bedside test, such as a drinking a sip of water; and any dysphagia assessment, defined as a more systematic examination performed by a speech pathologist/therapist or qualified clinician, according to local standard protocols. Only data pertaining to the first performed dysphagia screen/assessment were recorded. In practice, patients should have a dysphagia assessment after failing a dysphagia screen, ${ }^{12}$ and the results used to inform a local treatment plan to prevent aspiration pneumonia. As this was a pragmatic study, the specific practitioner, tool and approach to any dysphagia screen/assessment, were not specified in the protocol. Moreover, the study protocol allowed some flexibility in the assigned head position: to address any potential investigator concerns over harms, those patients allocated to the lying-flat position could be turned to their side; and patients assigned to either head position could have this interrupted for short intervals ( $\leq 3$ non-consecutive periods for $<30 \mathrm{~min}$ ) for feeding or mobility over the required 24 hours of applying the intervention, if it 
was considered necessary.

\section{Outcome}

The primary outcome for these analyses was pneumonia, reported as a serious adverse event, and classified according to the following pre-defined criteria: ${ }^{17}$ 'definite' pneumonia included $\geq 3$ features of new or worsening cough, increased respiratory rate, oxygen desaturation, fever $>38^{\circ}$ Celsius, leukocytosis or leukopenia, and purulent secretions, rales or bronchial breath sounds over the chest together with positive radiological abnormalities (patchy infiltration, lobar consolidation or pleural effusion); 'probable’ pneumonia was defined as $\geq 3$ of the listed features but without any radiological abnormalities, and; 'uncertain' pneumonia was $<3$ features with or without an abnormal X-ray. The secondary outcome was death or disability, defined as scores 3-6 on the modified Rankin scale (mRS) on blinded assessment at 90 days post-randomisation.

\section{Statistical analysis}

Both individual and hospital baseline characteristics were assessed in univariate analyses. Predictors of dysphagia screening (or assessment) and the outcomes of interest were determined by Chi-square test for categorical variables, t-test for approximately normally distributed variables, and Wilcoxon rank-sum test for skewed continuous variables. Variables identified with p values $<0.2$ were included in multivariable models. All potentially significant predictors were included in multilevel logistic regression models to estimate associations (Supplemental Table S1-S2). The primary analyses were the associations between use of a dysphagia screen (as a quality of stroke care performance measure, yes vs. no) and its result (fail vs. pass) on the outcomes of pneumonia and death or disability (mRS 3-6), independent of having a dysphagia assessment. Secondary analyses were for associations of a dysphagia assessment according to dysphagia screen status (unscreened, pass, and fail) and clinical outcomes. A complete case 
dataset was used to build models for analysing each of the association. The term 'unadjusted' was used in an initial, binomial logistic regression, hierarchical mixed model, where adjustment was made for the study design with fixed effects of head position (lying-flat vs. sitting-up) and crossover period, and random effects of cluster and interaction between cluster and crossover period. Sequential multi-level models were then 'adjusted', first for region of recruitment, and then with the addition of pre-specified baseline covariates and hospital characteristics. A further analysis was conducted to explore the influence of feeding restrictions on clinical outcomes in patients who either failed screening or assessment. Any interactions between significant variables and dysphagia screen/assessment were checked in each level of the models, and only those that were significant $(P<0.01)$ were included in the final model. Associations between exposures and outcomes were assessed across the pre-defined subgroups of the main trial. Multiple imputation was used for sensitivity analysis due to $12 \%$ missing data on 90 -day clinical outcome. Ten imputed datasets were generated and the odds ratio were pooled from the imputation analysis. Data are reported with odds ratios (OR) and 95\% confidence intervals (CI), and a standard level of significance was used $(P<0.05)$. No adjustments were made for multiplicity or missing data. All analyses were performed with SAS Software version 9.3 (SAS Institute, Cary, NC).

\section{Results}

Frequency and time of dysphagia screen and assessment

Among 11093 HeadPoST participants, there were 15 patients without any details on the use of dysphagia screening nor assessment. Overall, there were 8784 (79.2\%) and 3917 (35.3\%) patients who had a screen and assessment for dysphagia, respectively, but the frequency and timing for each varied significantly across regions (Table 1). Frequency of dysphagia screening was low in China (69.2\%) and South America (61.5\%) compared to Australia/UK (91.4\%) and India/Sri Lanka (87.0\%). Conversely, the frequency of dysphagia assessment was highest in 
South America (62.3\%) compared to the other regions (range, 23.3\% to 35.5\%). Overall, median times from admission to dysphagia screen and assessment were 2.2 hours (IQR 0.8-6.3) and 12.5 hours (IQR 1.8-28.0), respectively, but this varied from approximately 1 to 38 hours across regions, being shortest in China and longest in South America (Table 1). The majority of dysphagia assessments were undertaken subsequent to dysphagia screening; only $3 \%$ had an assessment recorded before a dysphagia screen.

Table 1. Frequency and timing of dysphagia screening and assessment in 11,093 stroke patients, by region of recruitment

\begin{tabular}{|c|c|c|c|c|c|c|c|c|}
\hline \multirow[b]{3}{*}{ Region } & \multicolumn{4}{|c|}{ Dysphagia screen performed } & \multicolumn{4}{|c|}{$\begin{array}{c}\text { Dysphagia assessment } \\
\text { performed }\end{array}$} \\
\hline & & & $\begin{array}{c}\text { Time from } \\
\text { hospital arrival }\end{array}$ & & & & $\begin{array}{l}\text { Time from } \\
\text { hospital arrival }\end{array}$ & \\
\hline & $\begin{array}{l}\mathrm{N} \\
(\%)\end{array}$ & $P$ value $^{\mathrm{a}}$ & $\begin{array}{c}\text { Median (IQR), } \\
\text { hrs }\end{array}$ & $P$ value $^{\mathrm{b}}$ & $\begin{array}{l}\mathrm{N} \\
(\%)\end{array}$ & $P$ value $^{\mathrm{a}}$ & $\begin{array}{l}\text { Median (IQR), } \\
\text { hrs }\end{array}$ & $P$ value ${ }^{\mathrm{b}}$ \\
\hline $\begin{array}{l}\text { Overall } \\
(\mathrm{N}=11,093)\end{array}$ & $8784(79.2)$ & & $2.2(0.8-6.3)$ & & $\begin{array}{c}3917 \\
(35.3)\end{array}$ & & $12.5(1.8-28.0)$ & \\
\hline \multicolumn{9}{|l|}{ Region } \\
\hline $\begin{array}{l}\text { Australia/UK } \\
(\mathrm{N}=4761)\end{array}$ & $4338(91.4)$ & $<0.0001$ & $2.6(1.1-5.7)$ & $<0.0001$ & $\begin{array}{c}1684 \\
(35.5)\end{array}$ & $<0.0001$ & $20.0(6.2-34.1)$ & $<0.0001$ \\
\hline $\begin{array}{l}\text { China }^{\mathrm{c}} \\
(\mathrm{N}=4652)\end{array}$ & $3218(69.2)$ & & $1.2(0.5-3.4)$ & & $\begin{array}{c}1488 \\
(32.0)\end{array}$ & & $1.4(0.7-4.4)$ & \\
\hline $\begin{array}{l}\text { India/Sri Lanka } \\
(\mathrm{N}=770)\end{array}$ & $670(87.0)$ & & $6.5(1.5-19.0)$ & & $\begin{array}{c}179 \\
(23.3)\end{array}$ & & $19.0(6.8-48.0)$ & \\
\hline $\begin{array}{l}\text { South America } \\
(\mathrm{N}=910)\end{array}$ & $558(61.5)$ & & $26.7(12.8-46.6)$ & & $\begin{array}{c}566 \\
(62.3)\end{array}$ & & $37.5(20.9-54.2)$ & \\
\hline
\end{tabular}

${ }^{a} \mathrm{P}$ value obtained from Chi-square test.

${ }^{\mathrm{b}} \mathrm{P}$ value obtained from Mann Whitney Test (Wilcoxon rank sum test).

cincludes Taiwan.

Baseline and hospital characteristics by screening and assessment

Patients without a dysphagia screen were younger, had greater pre-morbid disability, and more severe neurological impairment at the time of presentation (Supplemental Table S3). At hospital level, patients from hospitals with a stroke unit, guidelines for acute stroke treatment, local 
special pathways from stroke care, local protocols for swallow dysfunction, and speech pathologists, were more likely to receive screening (Supplemental Table S4). In comparison, in hospital with available protocol for swallow dysfunction, neurologists, dysphagia specialist nurses and speech pathologists, patients were more likely to have further dysphagia assessment (Supplemental Table S5).

\section{Results of dysphagia screen and further assessment}

Overall, 22.8\% (2004/8784) of patients failed a dysphagia screen (Table 2; Supplementary Figure S1). Compared to those who passed, dysphagia screen-fail patients were significantly older, with greater pre-morbid disability, cardiovascular disease and chronic obstructive pulmonary disease, and more severe baseline neurological impairment (Table 2). Of the 6778 patients who passed the dysphagia screen, 1775 (26.2\%) proceeded to a dysphagia assessment, which was passed by the great majority (96.1\%). There were 2292 patients who did not have a dysphagia screen, of whom 739 (32.2\%) proceeded to a dysphagia assessment (Supplemental Figure S1). They were older, more often female, with greater pre-morbid dependency and more severe baseline neurological impairment without coma, and more often placed on a feeding restriction regime, compared to those without a dysphagia assessment (Supplemental Table S6). Of the 2003 dysphagia screen-fail patients, there were 1402 (70.0\%) who proceeded to a dysphagia assessment; they tended to have milder neurological impairment compared to the 601 patient who did not have a dysphagia assessment (Supplemental Table S7). Patients who neither had a screen nor assessment were younger, had lower NIHSS scores, were less dependent and free of prior medical history, but with higher GCS scores at baseline (Supplemental Table S8).

Table 2. Baseline characteristics of patients, by result of dysphagia screen 


\begin{tabular}{lccc}
\hline & Pass & Fail & \\
Baseline characteristics & $\mathrm{N}=6778$ & $\mathrm{~N}=2004$ & $P$ Value \\
\hline Age, years & $66.9 \pm 13.6$ & $72.5 \pm 14.1$ & $<0.0001$ \\
Male & $4215(62.2)$ & $1062(53.0)$ & $<0.0001$ \\
Pathological subtype & & & \\
Acute ischaemic stroke & $5749(85.0)$ & $1715(86.0)$ & $<0.0001$ \\
Intracerebral haemorrhage & $492(7.3)$ & $211(10.6)$ & \\
Uncertain & $523(7.7)$ & $69(3.5)$ & \\
GCS score & $15(15-15)$ & $14(11-15)$ & $<0.0001$ \\
Severe (3-8) & $67(1.0)$ & $117(5.8)$ & $<0.0001$ \\
NIHSS score & $4(2-6)$ & $11(6-18)$ & $<0.0001$ \\
Severe $\geq 15$ & $258(3.8)$ & $705(35.2)$ & $<0.0001$ \\
Pre-stroke mRS score & & & $<0.0001$ \\
Independent (0-1) & $5504(81.2)$ & $1474(73.6)$ & \\
Mild disability but independent (2) & $676(10.0)$ & $206(10.3)$ & \\
Disabled (3-5) & $590(8.7)$ & $317(15.8)$ & \\
Prior cardiovascular disease ${ }^{\mathrm{a}}$ & $3373(49.8)$ & $1137(56.7)$ & $<0.0001$ \\
Prior COPD & $238(3.5)$ & $91(4.6)$ & 0.030 \\
Time to screen, hrs & $2.0(0.8-5.8)$ & $2.7(1.0-10.5)$ & $<0.0001$ \\
>24 hrs & $405(6.5)$ & $262(14.0)$ & $<0.0001$ \\
Feeding restrictions & $750(11.2)$ & $1681(84.1)$ & $<0.0001$ \\
\hline
\end{tabular}

Data are $n(\%)$, mean $\pm S D$, or median (interquartile range).

GCS denotes Glasgow coma scale, NIHSS National Institute of Health Stroke Scale, COPD chronic obstructive pulmonary disease.

aincludes history of heart disease, stroke or diabetes mellitus 


\section{Pneumonia outcome}

Overall, 362 (3.3\%) patients developed pneumonia, but the frequency varied significantly across regions and according to the use, and results of, a dysphagia screening and assessment (Supplemental Table S9). In particular, the frequency of pneumonia was higher in those who had dysphagia screen (or assessment), and especially in those who failed, and it was also associated with longer times to having a dysphagia screen (and assessment) (Supplemental Tables S9 and S10).

In multivariable analysis adjusted both individual and hospital level of characteristics, there was no association between the use of dysphagia screen and pneumonia (adjusted odds ratio [aOR] 1.20, 95\% CI 0.82-1.75; Figure 1A). However, compared to those who passed a dysphagia screen, screen-fail patients had a significantly higher risk of pneumonia $(1.5 \%$ vs. 10.0\%; aOR 3.00, 95\%CI 2.19-4.10) (Supplemental Table S9; Figure 2A, Model 2). Similarly, there was no association between use of dysphagia assessment and risk of pneumonia (Supplemental Figure S2A).

\section{[Insert Figure 1.]}

Death and dependency at 90-day

There were 12\% (1345/11093) of patients with missing 90-day clinical outcome data, who were younger and with greater pre-morbid disability compared to those with complete data (Supplemental Table S12). There was no association between use of dysphagia screen itself and poor clinical outcome (death or disability) (aOR 0.96, 95\%CI 0.81-1.13; Figure 1B). However, there was a significant association between those who failed compared to those who passed a dysphagia screen (68.1\% vs. 30.8\%, $P<0.0001$; aOR 1.66, 95\%CI 1.41-2.95; Supplemental Table S and Figure 2B). Compared to patients who did not have a dysphagia assessment, those who did had a higher risk of poor outcome (47.5\% vs. $34.6 \%, P<0.0001$; 
Supplemental Table S9) and there was a significant association between dysphagia assessment and poor outcome in patients who passed a dysphagia screen (aOR 1.39, 95\%CI 1.14-1.69; Supplemental Figure S2B). The significance of this association varied by region (Supplemental Figure S3). Failing a dysphagia assessment was significantly associated with increased risks of pneumonia (aOR 3.04, 95\% CI 2.11-4.39) and poor outcome (aOR 2.22, 95\% CI 1.76-2.80; Supplemental Figure S4).

\section{[Insert Figure 2.]}

\section{Subgroup analysis}

There was consistency in the relation between the use of dysphagia screening and pneumonia and poor outcome across patient subgroups (Supplemental Figures S5 and S6). Although there was no influence of head positioning on pneumonia, there was a lower risk of poor outcome in patients who were allocated to the lying-flat position (aOR 0.88, 95\%CI 0.77-1.00; Supplemental Figure S7). Similarly, there was no heterogeneity across subgroups in the results according to either a pass or fail on a dysphagia screen on pneumonia and poor outcome (Supplemental Figures S8 and S9). The effect of failing dysphagia screen is also consistent in patients with different head positions of the patients (Supplemental Figure S10B).

\section{Influence of feeding restrictions}

Patients who failed dysphagia screen were more likely to be placed on feeding restrictions compared to those passed ( $84.1 \%$ vs. $11.2 \%$, $\mathrm{p}<0.0001$; Table 2 ). The incidence of pneumonia and poor outcome were more in patients had feeding restrictions compared to those did not (9.5\% vs. $0.9 \%, \mathrm{p}<0.0001$ and $67.6 \%$ vs. $28.7 \%$, $\mathrm{p}<0.0001$, respectively; Supplemental Table S9). In the stratified analysis, subsequent use of feeding restrictions were related to higher risk of pneumonia, especially for patients failed a dysphagia screen or dysphagia assessment $(11.9 \%$ vs. 1.8\%, $\mathrm{p}<0.0001$; Supplemental Table S12 and aOR 4.06, 95\% CI 1.72-9.54; Figure S11A). 
There was considerable regional variation in the feeding regimes provided to patients who failed a dysphagia screen, although use of a nasogastric tube was the most common method (Supplemental Table S13).

\section{Sensitivity analysis}

With adjustment of both baseline and hospital characteristics, and use of multiple imputation for missing outcome, the results were similar to our primary analyses (Supplemental Figure S12-S13).

\section{Discussion}

In this large multinational study, we found no clear association between the use of a simple screen or detailed assessment of dysphagia regardless of the test results, as a quality of care measure, and either pneumonia or poor functional outcome after acute stroke. However, patients who failed either of these tests were clearly at increased risks of these adverse outcomes. The risk of pneumonia varied widely across regions and was related to the timing of dysphagia screen and assessment.

The overall 3\% frequency of pneumonia in our study was lower than reported in many other studies, ${ }^{15,17,18}$ but similar to that of a large registry study. ${ }^{19}$ The patients in our study were likely subjected to rigorous assessment and management of dysphagia by virtue of their participation in a clinical trial, where pneumonia was an expected adverse outcome. However, it is likely that selection bias and variable definitions influence the detection and reporting of pneumonia across studies. As in a real-life registry-based study, our protocol included consecutive patients with acute stroke. However, we did not specify any particular procedures for investigators to follow, and the assessment and management of dysphagia was performed according to local protocols. 
Dysphagia screening, assessment and management varies across countries in the context of specifications and interpretation of guidelines for stroke management. ${ }^{9-12}$ In our study, the median times from admission to screening and assessing dysphagia in Australia and UK were similar to another study conducted during 2013-2014. ${ }^{15}$ In China, dysphagia screens and assessments were performed at approximately the same time, although guidelines in this country make no specific recommendation regarding when or how to conduct them. ${ }^{10}$ Another UK stroke registry study has shown an association between delayed screen and assessment of dysphagia and increased risk of pneumonia. ${ }^{15}$ In our study, dysphagia screening was most delayed in South America, which may in part explain the higher rate of pneumonia (6.5\%) there compared to other regions.

We found no evidence of an association between the use of dysphagia screening and the risk of adverse outcomes. Another cluster clinical trial also showed no association of dysphagia screening and risk of pneumonia but rather a relation with lower risk of death and severe disability, ${ }^{13}$ which may have been due to an effect of other components of the care bundle targeting fever and hyperglycaemia. However, our study shows that patients who fail a dysphagia screen are at increased risk of pneumonia and poor clinical outcome, which is consistent with other studies. ${ }^{13,14,21-22}$ The majority (84\%) of patients who failed dysphagia screening were placed on feeding restrictions. These data are consistent with guideline recommendation for routine use of dysphagia screening in patients with acute stroke, with subsequent use of feeding restrictions or early dysphagia treatment in those who fail.

Another finding from our study was the increased risk of pneumonia or poor clinical outcome in 'screen-fail' or 'assessment-fail' patients and particularly those placed on restricted feeding. We assume it might be related to the mixed methods of feeding practices we measured, some of which might introduce adverse effect. A previous retrospective study has shown that the presence of nasogastric feeding was associated with reduced functional recovery and increased 
mortality after stroke. ${ }^{23}$ However, there is randomised evidence of early use of nasogastric tubes in dysphagia patients and lower risk of adverse outcomes, ${ }^{23}$ while dysphagia therapy programs appear to reduce the risk of pneumonia in the acute phase of stroke. ${ }^{8}$ We were unable to assess for any association of individual feeding actions on outcomes, as patients were often on multiple feeding restriction regimes. It is likely that analyses are complicated by indication bias, where high risk patients receive the intervention of interest, as our stratified analyses showed that patients who passed a dysphagia screen but subsequently had a dysphagia assessment, had higher risks of poor clinical outcome compared to other patients. Further studies concentrate on evaluating different methods of feeding actions subsequently after failing dysphagia screen, will be essential to improve patient’s clinical outcomes.

In our study, it is interesting to note that a quarter of patients passed a dysphagia screen yet went on to also receive a dysphagia assessment and majority (96\%) of them were reconfirmed as passed. Some of them may have deteriorated after screen and therefore, required further assessment; however, many may not have. This duplicated assessing for dysphagia was also noted in the QASC trial, with similar proportion (97\%) also deemed safe to swallow by the speech pathologist. ${ }^{13}$ Further examination of the reason for double swallowing surveillance in stroke patients is warranted. From another perspective, such inefficient duplicated assessment is costly and time-consuming, especially for some low-resourced settings. We recommend patients who had passed a screen and with no further deterioration should not be reviewed by a health professional.

We acknowledge several limitations, including the inability to pre-specify (or standardise) the methods of screening and assessment used across participating centres. As such, we were unable to provide any details regarding the type and quality of screening and assessments approaches undertaken for dysphagia, and for other aspects of background management. Another factor is that participants in our study are likely to have received a greater attention to 
standard of care processes including dysphagia monitoring and feeding actions because of the specific nature of our clinical trial assessing the influence of head positioning on stroke outcomes. However, this was a pre-specified secondary analysis of a large international trial based on local protocol by regions. Our findings reflect usual practice according to current guideline recommendations across countries. A major strength of our study is the large sample size of patients with a broad range of characteristics from a range of healthcare settings with variable resourcing levels. Moreover, selection bias was likely reduced compared to most conventional individual patient randomised clinical trials, by the inclusion of consecutive stroke patients within a cluster crossover design. We also considered the influences of institutional factors in multi-level models that included adjustment for various hospital characteristics.

\section{Conclusions}

The utility of dysphagia screening and assessment varies according to countries and local guidelines. Failing a dysphagia screen was associated with higher risk of pneumonia and poor outcome from acute stroke. Subsequent feeding restrictions is related to increased risk of adverse outcomes. Further RCTs that evaluating the effects of feeding actions are urgent to improve patient's outcome.

\section{Acknowledgements}

We thank the participants and investigators of the Head Positioning in Acute Stroke Trial (HeadPoST) study.

\section{Contributors}

CSA, HA, MH, CW and LS contributed to the concept and rationale for the study. MO did the statistical analysis with assist from KR. MO wrote the first draft of manuscript with input from CSA. All authors have seen and approved the final version of the manuscript for publication.

\section{Disclosures}


PML reports grants from The George Institute for Global Health and Clínica Alemana de Santiago, during the conduct of the study; and non-financial support from Boehringer Ingelheim, grants and personal fees from Bayer and AstraZeneca, and grants from CONICYT, outside the submitted work. MLH holds a National Health and Medical Research Council of Australia (NHMRC) Career Development Fellowship. PMV reports grants from Clínica Alemana de Santiago, during the conduct of the study; and grants from CONICYT, outside the submitted work. PMPN received grants for the Brazilian Stroke Research Network by CNPQ (402388/2013-5) for conduct this study. TGR is an National Institutes of Health Research (NIHR) Senior Investigator. CSA holds an NHMRC Senior Principal Research Fellowship, and reports honoraria and travel reimbursement from Takeda, Boehringer Ingelheim and Amgen outside of this study. The other authors have no disclosures to report. 


\section{Reference}

1. Koennecke HC, Belz W, Berfelde D, Endres M, Fitzek S, Hamilton F, et al. Factors influencing in hospital mortality and morbidity in patients treated on a stroke unit. Neurology 2011; 77: 965-972.

2. Hannawi Y, Hannawi B, Rao CP, Venkatasubba, Suarez J, Bershad EM. Stroke associated pneumonia: major advances and obstacles. Cerebrovascular Dis 2013; 35: 430-443.

3. Katzan L, Dawson V, Thomas L, Votruba E, Cebul D. The cost of pneumonia after acute stroke. Neurology 2007; 68: 1938-1943.

4. Rofes L, Arreola V, Almirall J, et al. Diagnosis and management of oropharyngeal dysphagia and its nutritional and respiratory complications in the elderly. Gastroenterol Res Pract 2011; 2011:1-13. https://doi.org/10.1155/2011/818979

5. Titsworth LW et al. Prospective quality initiative to maximize dysphagia screening reduces hospital-acquired pneumonia prevalence in patients with stroke. Stroke 2013 44:3153160.

6. Joundi RA, Martino R, Saposnik G, Giannakeas V, Fang J, Kapral MK. Predictors and outcomes of dysphagia screening after acute ischemic stroke. Stroke 2017; 48: 900-906.

7. Cohen DL, Roffe C, Beavan J, Blackett B, Fairfield CA, Hamdy S, et al. Post-stroke dysphagia: a review and design considerations for future trials. Int J Stroke 2016; 11: 399411.

8. Smith EE, Kent DM, Bulsara R, Ketan LY, Lichtman JH, Reeves JM et al. Effect of dysphagia screening strategies on clinical outcomes after stroke. Stroke 2018; 49: e123e128.

9. Indian Stroke Association Consensus Statement. Recommendations for the early management of acute ischemic stroke a consensus statement for healthcare professionals 
from the India Stroke Association, 2014. https://www.stroke-india.org/stroke.pdf. Accessed June 24, 2018.

10. Chinese Cerebrovascular Association. Chinese guidelines for acute ischemic stroke treatment and management 2014. Chinese J Neurology 2015; 48: 246-248.

11. Scottish Intercollegiate Guidelines network. Guideline 119: management of patients with stroke: identification and management of dysphagia a national guideline, 2011. http://www.sign.ac.uk/assets/sign119.pdf. Accessed June 24, 2018.

12. Stroke Foundation Australia. Chapter 3 of 8: Acute medical and surgical management: Dysphagia. Clinical guidelines for stroke management 2017. https://www.magicapp.org/app\#/gMOUYuideline/2280. Accessed June 29, 2018.

13. Middleton S, McElduff P, Ward J, Grimeshaw JM, Dale S, D’Este C et al. Implementation of evidence-based treatment protocols to manage faver, hyperglycaemia, and swallowing dysfunction in acute stroke (QASC): a cluster randomised controlled trial. Lancet 2011; 378: 1699-1706

14. Palli C, Fandler S, Doppelhofer K, Niederkorn K, Enzinger C, Vetta C, Trampusch E, Schmidt R, Fazekas F, Gattringer T. Early dysphagia screening by trained nurses reduces pneumonia rate in stroke patients: a clinical intervention study. Stroke 2017; 48: 25832585.

15. Bray BD, Smith CJ, Cloud GC, Enderby P, James M, Paley L, Tyrrell PJ, Wolfe CDA, Rudd AG. The association between delays in screening for and assessing dysphagia after acute stroke and the risk of stroke associated pneumonia. J Neurol Neurosurg Psychiatry 2017; 88: 25-30

16. Anderson CS, Arima H, Lavados P, Billot L, Hackett ML, Olavarria VV et al. Clusterrandomized, crossover trial of head positioning in acute stroke. New Engl J Med 2017; 376: 2437-47. 
17. Smith CJ, Kishore AK, Vail A, et al. Diagnosis of stroke-associated pneumonia: recommendations from the pneumonia in stroke consensus group. Stroke 2015; 46: 23352340

18. Finlyson O, Kapral M, Hall R, Asllani E, Selchen D, Saposnik G. risk factors, inpatients care, and outcomes of pneumonia after ischemic stroke. Neurology 2011; 77: 1338-1345.

19. Lakshminarayan K, Tsai AW, Tong X, Vazquez G, Peacock JM, George MG, Luepker RV, Anderson DC. Utility of dysphagia screening results in predicting poststroke pneumonia. Stroke 2010; 41: 2849-2854.

20. Hichey JA, Shephard T, Furie K, Smith D, Wang D, Tonn, S. Formal dysphagia screening protocols prevent pneumonia. Stroke 2005; 36: 1972-1976.

21. Joundi RA, Martino R, Saposnik G, Giannakeas, V, Fang J, Kapral MK. Predictors and outcomes of dysphagia screening after acute ischemic stroke. Stroke 2017; 48: 900-906.

22. Geeganage C, Beavan J, Ellender S, Bath PM. Interventions for dysphagia and nutritional support in acute and subacute stroke. Cochrane Database Systematic Review 2012; 10: CD000323. Doi: 10.1002/14651858. CD000323. Pub2.

23. Schwarz M, Coccetti A, Murdoch A, Cardell E. The impact of aspiration pneumonia and nasogastric feeding on clinical outcomes in stroke patients. J Clin Nursing 2018; 27: e235e241 
